\title{
Comparative efficacy between monoclonal antibodies and conventional drugs in postmenopausal women with osteoporosis: a network meta-analysis
}

\author{
Qingtao Jiang', Feng Zhang ${ }^{2}$, Baoli Zhu' ${ }^{2}$, Hengdong Zhang ${ }^{2}$, Lei Han ${ }^{2}$, Xin Liu $^{2}$ \\ ${ }^{1}$ Department of Medicine, Jiangsu Health Vocational College, Nanjing, China; ${ }^{2}$ Institute of Occupational Disease Prevention, Jiangsu Provincial \\ Center for Disease Control and Prevention, Nanjing, China \\ Contributions: (I) Conception and design: X Liu, Q Jiang; (II) Administrative support: B Zhu, H Zhang; (III) Collection and assembly of data: Q Jiang, \\ F Zhang, L Han; (IV) Data analysis and interpretation: Q Jiang, X Liu; (V) Manuscript writing: All authors; (VI) Final approval of manuscript: All \\ authors; (VII) Final approval of manuscript: All authors. \\ Correspondence to: Xin Liu. Institute of Occupational Disease Prevention, Jiangsu Provincial Center for Disease Control and Prevention, No. 122 \\ Maihua Road, Nanjing, China. Email: liuxinjsha@163.com.
}

\begin{abstract}
Background: To evaluate the efficacy of different pharmacological interventions, with emphasis on monoclonal antibodies (McAbs) for the treatment of postmenopausal osteoporotic women.

Methods: A search of PubMed, Google Scholar, Embase, and the Cochrane Library, as well as China National Knowledge Infrastructure (CNKI) was performed. Data were collected and pooled using Bayesian network meta-analysis (NMA), which conducts both direct and indirect comparisons. The primary outcome was the percentage change in bone mineral density (BMD) in the lumbar spine from baseline to 1 year of treatment. All drugs were ranked based on the surface under the cumulative ranking area (SUCRA). Furthermore, the heterogeneity, consistency, and publication bias of the enrolled literature were assessed.

Results: There were 14 randomized controlled trials (RCTs) consisting of 4,881 participants included to compare 11 interventions. Compared with that of a placebo, all the 10 selected therapies showed significant efficacy through changes in BMD ranging from $6.0 \%$ to $19.0 \%$ (all $\mathrm{P}<0.01$ ). As the SUCRA values indicated, the therapeutic performance of antibody drugs was better than that of the conventional chemical agents, and blosozumab was the best choice (SUCRA 99.2\%) to improve lumbar spine BMD.

Conclusions: Novel McAb treatments achieved better therapeutic effects in the treatment of postmenopausal osteoporosis (PMO), especially blosozumab. Further trials are needed to investigate and optimize the delivery strategy of McAbs.
\end{abstract}

Keywords: Postmenopausal osteoporosis (PMO); monoclonal antibody (McAb); network meta-analysis (NMA)

Submitted Jun 22, 2020. Accepted for publication Oct 22, 2020.

doi: 10.21037/apm-20-1294

View this article at: http://dx.doi.org/10.21037/apm-20-1294

\section{Introduction}

Because of its prevalence worldwide, osteoporosis is considered a serious public health concern that usually decreases the quality of life because of fracture-induced disability (1). Currently, it is estimated that more than 200 million people suffer from this disease worldwide. Although osteoporosis can strike at any age, it is most common among older people. Approximately $30 \%$ of all postmenopausal women with estrogen withdrawal have osteoporosis in the United States and Europe. The prevalence of osteoporosis in men over 50 years of age in China $(6.0 \%)$ is not significantly different from that in other parts of the world $(5.1 \%$ in the USA, $6.6 \%$ in Canada, and $7.3 \%$ in Korea). However, the morbidity of osteoporosis in women in China varies considerably from those in the western countries $(32.1 \%$ vs. $16.5 \%$ in the USA and $15.8 \%$ 
in Canada) (2).

Osteoporosis is a preventable and treatable disease. Several prospective studies have suggested that improved bone mineral density (BMD) is associated with a reduction in the fracture rate $(3,4)$. Among the people not suffering from osteoporosis, the low bone mass proportion was $49.9 \%$ in males and $67.6 \%$ in females over 50 years old. Hence, improving $\mathrm{BMD}$ and reducing fractures are the primary therapeutic goals (5).

There are two main categories of conventional therapies applied to prevent or treat postmenopausal osteoporosis (PMO). One is anti-resorptive chemical agents containing estrogen or selective estrogen receptor modulators (bazedoxifene, raloxifene), calcitonin, bisphosphonates (alendronate, ibandronate, risedronate, and zoledronate), and odanacatib. The other category consists of the drugs that have anabolic effects on bones, such as strontium ranelate, teriparatide, and other similar drugs (6). With advances in biotechnology, the development of ever more sophisticated treatments employing novel mechanisms of action is rendered possible. As a molecular targeted therapy, fully humanized monoclonal antibodies (McAbs) against RANKL (denosumab) or sclerostin (romosozumab or blosozumab) have been put through large-scale clinical trials in succession, resulting in improvements of BMD and reductions in fracture risk (7-9).

With numerous choices, the best healthcare method for postmenopausal women with osteoporosis is still controversial. Though there are several courses of treatment for osteoporosis, including combinations of drugs, the proper selection of different therapies is a topic that remains open for debate. Unfortunately, there is still no synthesized evidence regarding how to improve therapeutic efficacy and reduce the physical burden resulting from drugs, especially whether novel McAb drugs are superior to the traditional chemical agents in women with PMO (10-12).

Previously, randomized controlled trials (RCTs) and traditional pairwise meta-analyses have been performed to determine the most effective therapy. However, the conclusions remain controversial, especially for the selection of McAb drugs. Because network meta-analysis (NMA) enables the ranking of all possible regimens, although without direct comparisons, we performed a systematic review and NMA to identify the efficacy of McAbs with other therapeutic agents for the treatment of osteoporosis $(13,14)$. We presented our study according to the Preferred Reporting Items for Systematic Reviews and MetaAnalyses (PRISMA) reporting checklist. The influence of
11 interventions (1, denosumab; 2 , denosumab + placebo; 3 , denosumab + teriparatide; 4 , romosozumab; 5 , blosozumab; 6, alendronate; 7, teriparatide; 8, zoledronic acid; 9, ibandronate; 10 , risedronate; and 11 , placebo) on the percent change in BMD from baseline to 12 months of treatment during which the lumbar spine was comprehensively assessed. We present the following article in accordance with the PRISMA reporting checklist (available at http://dx.doi. org/10.21037/apm-20-1294).

\section{Methods}

\section{Literature search}

This study was conducted according to the PRISMA protocols (15-19). We systematically searched PubMed, Google Scholar, Embase, and Cochrane Library, as well as China National Knowledge Infrastructure (CNKI), a key national research and information publishing institution in China, for articles up to August 31, 2020, with the restriction of language to English and Chinese. To fully identify studies covering relevant participants, the literature search involved the querying of the terms "Denosumab", "Romosozumab", "Blosozumab", "Osteoporosis", and "Randomized controlled trial (RCT)" conducted in humans. Additionally, reference lists of the identified RCTs were manually scanned to identify the related research in order to increase the recall and accuracy ratio. The electronic search procedure followed the PRISMA guidelines and used the PRISMA Extension for NMA.

\section{Study eligibility}

We collected all RCTs on the comparison of therapeutic effects containing McAb drugs for the treatment of PMO. Inclusion criteria of studies included those with (I) a study population of postmenopausal women with osteoporosis or low BMD, (II) a study design including therapeutic RCT, (III) the information on the interventions included McAbs (denosumab, romosozumab, or blosozumab), (IV) wherein all participants were required to take at least $1,000 \mathrm{mg}$ of elemental calcium and 400 IU of vitamin D daily as a basic treatment, and the dosing regimen of each $\mathrm{McAb}$ was identical (denosumab $60 \mathrm{mg}$; romosozumab $210 \mathrm{mg}$; blosozumab $270 \mathrm{mg}$ ). Studies without consistent doses or treatment courses, as well as the extraction of outcome data, were excluded. In this NMA, the available primary endpoint was the percent change in BMD of the lumbar spine at 


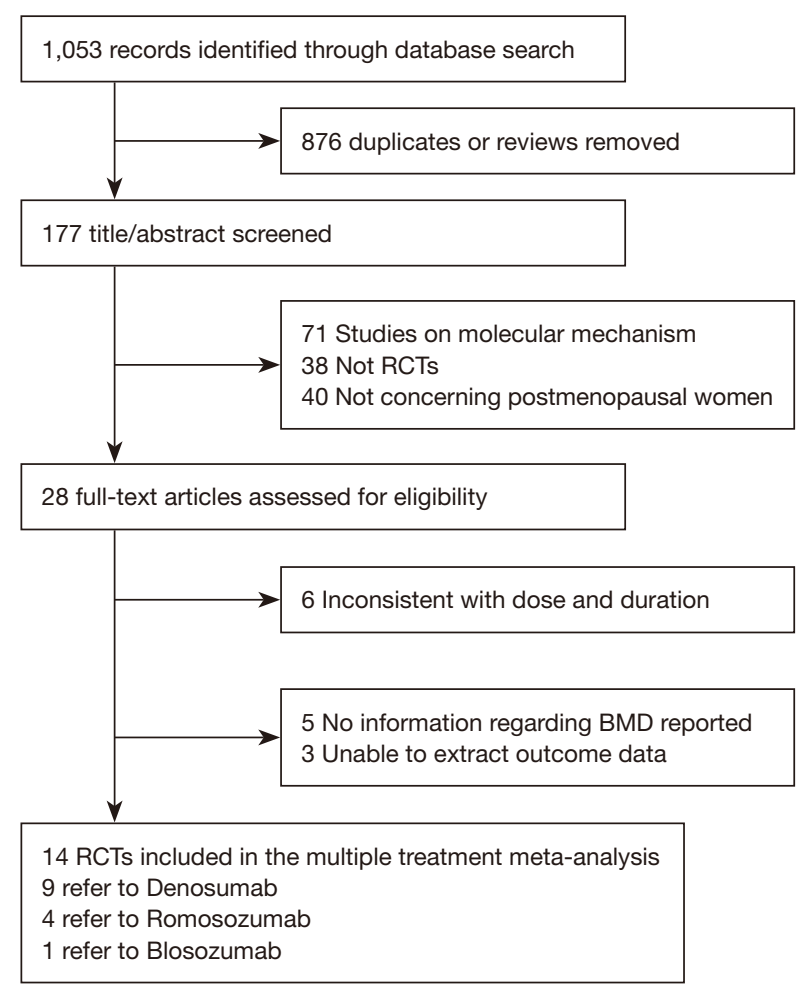

Figure 1 Study flow and selection diagram.

12 months. Percent change from baseline in BMD for the lumbar spine was synthetically assessed. Two investigators (X.L. and Q.J.) independently determined whether the trials met the inclusion criteria, with discrepancies resolved by consensus.

\section{Data extraction and quality assessment}

We assessed the risk of bias in contributing studies with standard criteria. Two authors (F.Z. and Q.J.) extracted data and evaluated quality, respectively, with a discussion with the third reviewer (X.L.) to resolve any discrepancies. The collected information included participant demographic information (age, sex) and study characteristics (authors, publication year, sample size, duration of intervention, and outcome). Regarding the lack of standard deviation (SD), the estimation method via sample size and confidence interval (CI) was adopted to deduce SD. Additionally, the quality of evidence was assessed using the Cochrane Risk of Bias (ROB) Tool, including the following seven items: (I) random sequence generation, (II) allocation concealment, (III) blinding of participants and personnel, (IV) blinding of outcome assessment, (V) incomplete outcome data, (VI) selective reporting, and (VII) other sources of bias.

\section{Data synthesis and statistical analysis}

The NMA was performed using R software 3.5.2 with the "Gemtc" package, which recalls JAGS in R for Markov chain Monte Carlo (MCMC) sampling. The mean \pm SD was utilized to compare different agents with respect to various clinical outcomes. We conducted a conventional metaanalysis for treatments that were directly compared in RCTs by the Bayesian random-effects model. Convergence was checked using the Brooks-Gelman-Rubin diagnostic and trace plots. A comparison-adjusted funnel plot was used to detect the presence of publication bias in the NMA. Ranking probabilities of all treatments used the surface under the cumulative ranking area (SUCRA). Inconsistencies between direct and indirect evidence in the network were evaluated using global and local inconsistency tests.

\section{Results}

\section{Study selection and characteristics}

Of the 1,053 citations screened, 14 studies (20-33) involving 4,881 subjects were recruited in our meta-analysis and the selection details are shown in Figure 1. The baseline characteristics and J Cochrane ROB evaluation of the included studies are presented in Table 1 and Figure S1. In 14 studies, nine trials investigated the efficacy of denosumab relative to other medications; four studies provided data on romosozumab elevation; and one study provided results on blosozumab. No direct comparison between these McAb agents was conducted.

\section{Model construction and publication bias}

A network plot of treatment comparisons on the percent change in BMD is shown in Figure 2. The nodes represent the interventions and their sizes represent the number of participants. Edges represent the availability of direct comparisons between pairs of interventions, whereas the width represents the number of studies comparing the pair of interventions.

For each analysis, we generated 5,000 simulations for each of the two sets of different initial values and discarded the first 2,000 simulations as the burn-in period. A total of 25,000 instances of iterations were increased to acquire 
Table 1 Characteristics of included studies and respective Jadad scoring

\begin{tabular}{|c|c|c|c|c|c|c|c|c|}
\hline ID & Author & Year & \multicolumn{3}{|c|}{ Intervention-1 } & \multicolumn{3}{|c|}{ Intervention-2 } \\
\hline 1 & Bolognese & 2013 & Denosumab & 232 & $73.0(4.8)$ & Placebo & 209 & $73.0(5.4)$ \\
\hline 2 & Kendler & 2010 & Denosumab & 243 & $66.9(7.8)$ & Alendronate & 238 & $68.2(7.7)$ \\
\hline \multirow[t]{2}{*}{4} & McClung & 2006 & Denosumab & 41 & $63.1(8.1)$ & Alendronate & 45 & $62.8(8.2)$ \\
\hline & & & & & & Placebo & 40 & $63.7(9.1)$ \\
\hline 5 & McClung & 2017 & Denosumab & 157 & $72.8(4.7)$ & Placebo & 128 & $72.3(5.4)$ \\
\hline \multirow[t]{2}{*}{9} & Leder & 2013 & Denosumab & 33 & $66.3(8.3)$ & $\begin{array}{c}\text { Denosumab + } \\
\text { teriparatide }\end{array}$ & 29 & $65.9(9.0)$ \\
\hline & & & & & & Teriparatide & 30 & $65.5(7.9)$ \\
\hline \multirow[t]{2}{*}{10} & Genant & 2017 & Romosozumab & 24 & $64.3(4.7)$ & Teriparatide & 30 & $65.8(5.7)$ \\
\hline & & & & & & Placebo & 27 & $66.1(5.8)$ \\
\hline 11 & Ishibashi & 2017 & Romosozumab & 59 & $68.3(5.9)$ & Placebo & 59 & $67.8(7.2)$ \\
\hline \multirow[t]{2}{*}{12} & Keaveny & 2017 & Romosozumab & 24 & $64.3(4.7)$ & Teriparatide & 28 & $65.8(5.7)$ \\
\hline & & & & & & Placebo & 27 & $66.1(5.8)$ \\
\hline
\end{tabular}

satisfactory convergence, as shown in the trace-density plots, as well as diagnostics plots, through which the potential scale reduction factor (PSRF) was obtained as 1.01 (Figures S2,S3). The publication bias is revealed by the funnel plot in Figure S4 and there was no apparent evidence of asymmetry.

\section{Efficacy outcome-percentage change of BMD}

The effects on the percent change in BMD were generated on the direct meta-analysis forest plots. Because no specific evidence among McAb drugs had been reported, this result was statistically drawn by network estimation via the pairwise comparisons between conventional drugs versus McAbs. The analysis suggested that romosozumab and blosozumab were significantly superior to other conventional interventions (Figure $3 A, B$ ), except for denosumab (Figure 3C).

In Figure S5, we illustrated the inconsistency in the seven triangular loops formed by the three treatments compared with each other using the method of moments. The upper $95 \%$ CI (truncated) of IF close to 0 indicated that the network was generally consistent where the direct and indirect effect sizes in the loop highly overlapped.

Probability sorting data after n.adapt $=5,000$ and n.iter $=20,000$ iterations is shown in Table 2, which consists of rows and columns equal to the total number of 


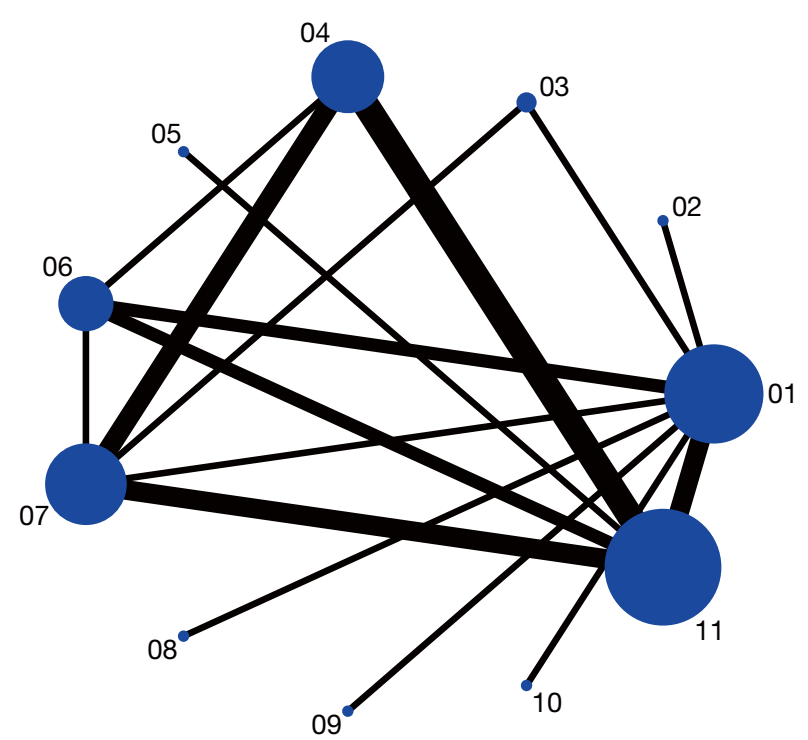

Figure 2 Evidence network of eligible comparisons of NMA. The width of lines indicates the number of trials, whereas the area of circles represents the cumulative number of patients for each intervention. NMA, network meta-analysis. interventions (11 in this study). It could be directly inferred that blosozumab was likely the best, whereas the placebo was the worst. However, the specific ranking requires needs further analysis. And Figure 4 is a probability sorting graph expressed by a histogram, which allowed the researchers to predict the best and worst intervention more quickly. Indeed, a similar order could be concluded as a comparison to that derived from the data table.

A cumulative ranking plot and SUCRA could aid researchers in decision making. Table 3 is the cumulative probability data, based on which the cumulative ranking plot of interventions was produced and SUCRA calculated. Figure 5 was the cumulative ranking probability diagram and the corresponding SUCRA value of each agent (underneath) through which the curative effect of interventions could be ranked. Blosozumab and romosozumab ranked first and second (SUCRA of $99.2 \%$ and $94.8 \%$, respectively), followed by denosumab combined with teriparatide (SUCRA of $83.0 \%$ ). Placebo was ranked as the least effective treatment (SUCRA of 7.1\%).

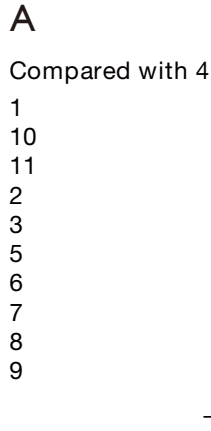

C

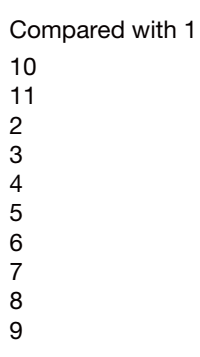

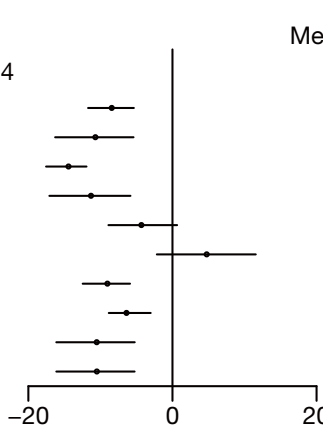

20

Mean Difference (95\% Crl)

$-7.9(-12 .,-5.4)$

$-10 .(-16 .,-5.4)$

$-14 .(-18 .,-12$.

$-11 .(-17 .,-5.8)$

$-3.8(-8.9,0.65)$

$5.2(-2.2,12$.

$-8.5(-12 .,-5.9)$

$-5.9(-8.8,-3.0)$

$-10 .(-16 .,-5.2)$

-10. $(-16 .,-5.3)$

Mean Difference $(95 \% \mathrm{Crl})$

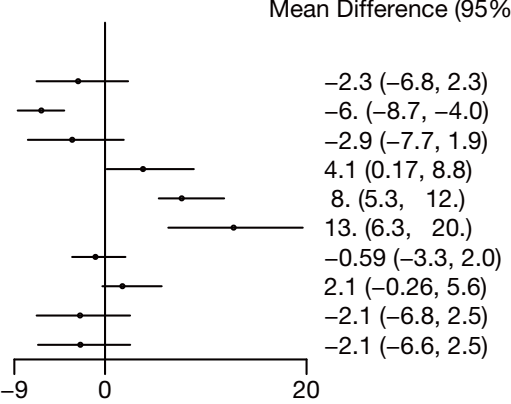

B

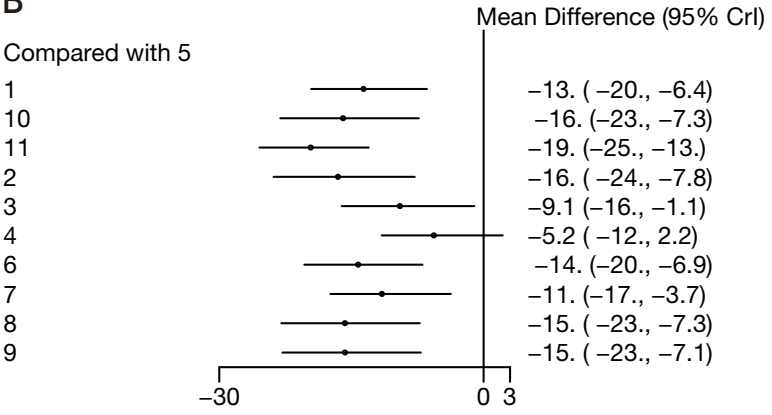

Figure 3 Forest plots for lumbar spine BMD compared between McAbs and conventional drugs. (A) Comparison of efficacy between romosozumab and other interventions; (B) comparison of efficacy between blosozumab and other interventions; (C) comparison of efficacy between denosumab and other interventions. BMD, bone mineral density; McAbs, monoclonal antibodies. 
Table 2 Probability sorting data for 11 interventions

\begin{tabular}{|c|c|c|c|c|c|c|c|c|c|c|c|}
\hline Ranks & \multicolumn{11}{|c|}{ Interventions } \\
\hline 1 & 0.00000 & 0.00075 & 0.00000 & 0.00067 & 0.00860 & 0.06247 & 0.92570 & 0.00002 & 0.00017 & 0.00078 & 0.00085 \\
\hline 2 & 0.00005 & 0.00213 & 0.00000 & 0.00160 & 0.04103 & 0.89363 & 0.05442 & 0.00032 & 0.00273 & 0.00217 & 0.00192 \\
\hline 3 & 0.00190 & 0.00840 & 0.00000 & 0.00723 & 0.80882 & 0.03892 & 0.01150 & 0.00415 & 0.10012 & 0.00945 & 0.00952 \\
\hline 5 & 0.56585 & 0.04702 & 0.00008 & 0.03465 & 0.02303 & 0.00085 & 0.00142 & 0.15640 & 0.06482 & 0.05253 & 0.05335 \\
\hline 6 & 0.27367 & 0.06418 & 0.00040 & 0.04595 & 0.01050 & 0.00028 & 0.00120 & 0.43505 & 0.02208 & 0.07270 & 0.07398 \\
\hline 7 & 0.10362 & 0.16387 & 0.00207 & 0.10358 & 0.00712 & 0.00015 & 0.00078 & 0.21080 & 0.01070 & 0.19598 & 0.20133 \\
\hline 8 & 0.02975 & 0.22147 & 0.00857 & 0.15430 & 0.00532 & 0.00005 & 0.00048 & 0.11215 & 0.00512 & 0.23512 & 0.22768 \\
\hline 11 & 0.00000 & 0.03442 & 0.84547 & 0.06055 & 0.00035 & 0.00000 & 0.00010 & 0.00060 & 0.00003 & 0.02855 & 0.02993 \\
\hline
\end{tabular}

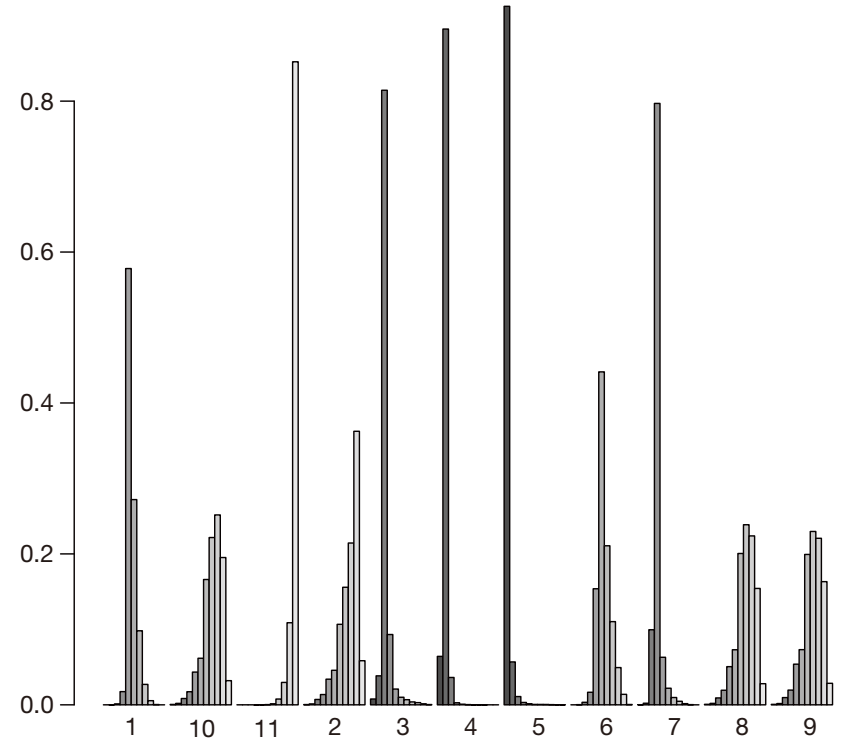

Figure 4 Probability ranking histogram of 11 interventions.

\section{Discussion}

In this NMA, we investigated the efficacy of McAbs and conventional interventions in postmenopausal women with osteoporosis. Through a systematic search, we found 14 RCTs recruiting 4,881 participants and observed the effect of monotherapy (denosumab, romosozumab, blosozumab, alendronate, teriparatide, zoledronic acid, ibandronate, risedronate, and placebo) and sequential or combined therapy (denosumab + placebo or denosumab + teriparatide) on increasing BMD of the lumbar vertebra. Overall, the high quality of the articles we chose, having neither major publication bias nor large inconsistencies, which could have contributed to the reliability of our research. As shown in our assessment, blosozumab was the best agent for the treatment of PMO based on the SUCRA, whereas the placebo had the lowest effectiveness among the 11 treatments.

From a clinical point of view, osteoporosis-related fractures most commonly occur in the hip, backbone (spine), and wrist. Back pain caused by a vertebral compression fracture in the spine is a typical sign and symptom in the initial stages of bone loss. As is well known, BMD is the most intuitive and reliable indicator reflecting the therapeutic effect of osteoporosis (34). Thus, we chose the percent change of lumbar spine BMD from baseline as our primary endpoint. No apparent relationship between tested drugs and adverse events was observed, except such complaints as the incidence of injection-site reactions or dyspepsia, which did not lead to discontinuation of the study drug or withdrawal from the study.

The resulting network model portrayed a picture of comparable outcomes in BMD change to select the optimum choice for PMO. Molecular targeting drugs initially became a hot topic in the field of cancer therapy, 
Table 3 Cumulative ranking data for 11 interventions

\begin{tabular}{|c|c|c|c|c|c|c|c|c|c|c|c|}
\hline $\begin{array}{l}\text { Cumulative } \\
\text { ranks }\end{array}$ & \multicolumn{11}{|c|}{ Interventions } \\
\hline 1 & 0.00000 & 0.00075 & 0.00000 & 0.00067 & 0.00860 & 0.06247 & 0.92570 & 0.00002 & 0.00017 & 0.00078 & 0.00085 \\
\hline 2 & 0.00005 & 0.00288 & 0.00000 & 0.00227 & 0.04963 & 0.95610 & 0.98012 & 0.00033 & 0.00290 & 0.00295 & 0.00277 \\
\hline 3 & 0.00195 & 0.01128 & 0.00000 & 0.00950 & 0.85845 & 0.99502 & 0.99162 & 0.00448 & 0.10302 & 0.01240 & 0.01228 \\
\hline 5 & 0.58722 & 0.07648 & 0.00008 & 0.05807 & 0.97225 & 0.99948 & 0.99697 & 0.17820 & 0.95985 & 0.08488 & 0.08652 \\
\hline 6 & 0.86088 & 0.14067 & 0.00048 & 0.10402 & 0.98275 & 0.99977 & 0.99817 & 0.61325 & 0.98193 & 0.15758 & 0.16050 \\
\hline 7 & 0.96450 & 0.30453 & 0.00255 & 0.20760 & 0.98987 & 0.99992 & 0.99895 & 0.82405 & 0.99263 & 0.35357 & 0.36183 \\
\hline 8 & 0.99425 & 0.52600 & 0.01112 & 0.36190 & 0.99518 & 0.99997 & 0.99943 & 0.93620 & 0.99775 & 0.58868 & 0.58952 \\
\hline 11 & 1.00000 & 1.00000 & 1.00000 & 1.00000 & 1.00000 & 1.00000 & 1.00000 & 1.00000 & 1.00000 & 1.00000 & 1.00000 \\
\hline
\end{tabular}

and gradually expanded to other areas of chronic diseases. Fortunately, McAbs could enhance drug efficiency by identifying targets, reduce the frequency of drug delivery, and improve patient compliance. In particular, $\mathrm{McAb}$ drugs were administered by subcutaneous (SC) injections delivering $60 \mathrm{mg}$ of denosumab every 6 months, $210 \mathrm{mg}$ of romosozumab every 3 months, and $270 \mathrm{mg}$ of blosozumab every 2 weeks. However, most conventional anti-osteoporosis drugs, such as a bisphosphonate, need to be taken daily or weekly. As shown in our study, regarding the optimal selection for postmenopausal osteoporotic women, the list was led by blosozumab, romosozumab, and denosumab combined with teriparatide, and strongly indicated the advantages of targeted antibody drugs.

The development of McAb agents is entirely based on a thorough understanding of the molecular mechanisms of bone formation and resorption. RANKL and sclerostin are key modulators in osteoclast and osteoblasts activity, respectively (35-37). Binding to RANKL (denosumab) or sclerostin (romosozumab or blosozumab) could specifically block bone resorption or promote bone formation $(38,39)$. Excitedly, the efficacy and safety evaluation of these McAbs was already accomplished in the phase III clinical trial. Additionally, denosumab (Trade name "Prolia") has already won final FDA approval, whereas romosozumab and blosozumab are under approval applications with the FDA $(40,41)$.

It is indisputable that the placebo was the most ineffective, but unexpectedly denosumab alone did not outperform other interventions, except for its combination with teriparatide, showing a significant improvement in BMD change in postmenopausal osteoporotic women. Currently, the application of denosumab has come to full maturity and is in universal clinical practice $(42,43)$. Thus, modified drug dosage or interval and drug combination may be the potential strategies in the future.

To our knowledge, this study has at least two advantages. First, our study is an early NMA with respect to the efficacy of McAb therapies for PMO. Second, we chose highquality research that was highly consistent in the project design methodology and outcome evaluation, without serious inconsistencies and small sample bias that could interfere in achieving reliable results. However, there are some limitations in our NMA. To avoid confounding factors and achieve comparability among studies, the selections of drug dosage and duration are quite consistent. Therefore, taking into consideration other endpoints, such as total hip or femoral neck, and additional indicators, such as serum biomarkers (P1NP and $\beta$-CTX) (44), a more comprehensive study and evaluation must be conducted, and long-term effects of McAbs should be confirmed in the future. Furthermore, as a newly developed agent, the number of RCTs on blosozumab is still limited. However, its clinical application is highly promising. Finally, for clinical practice, the findings should be interpreted carefully and be translated with caution. 

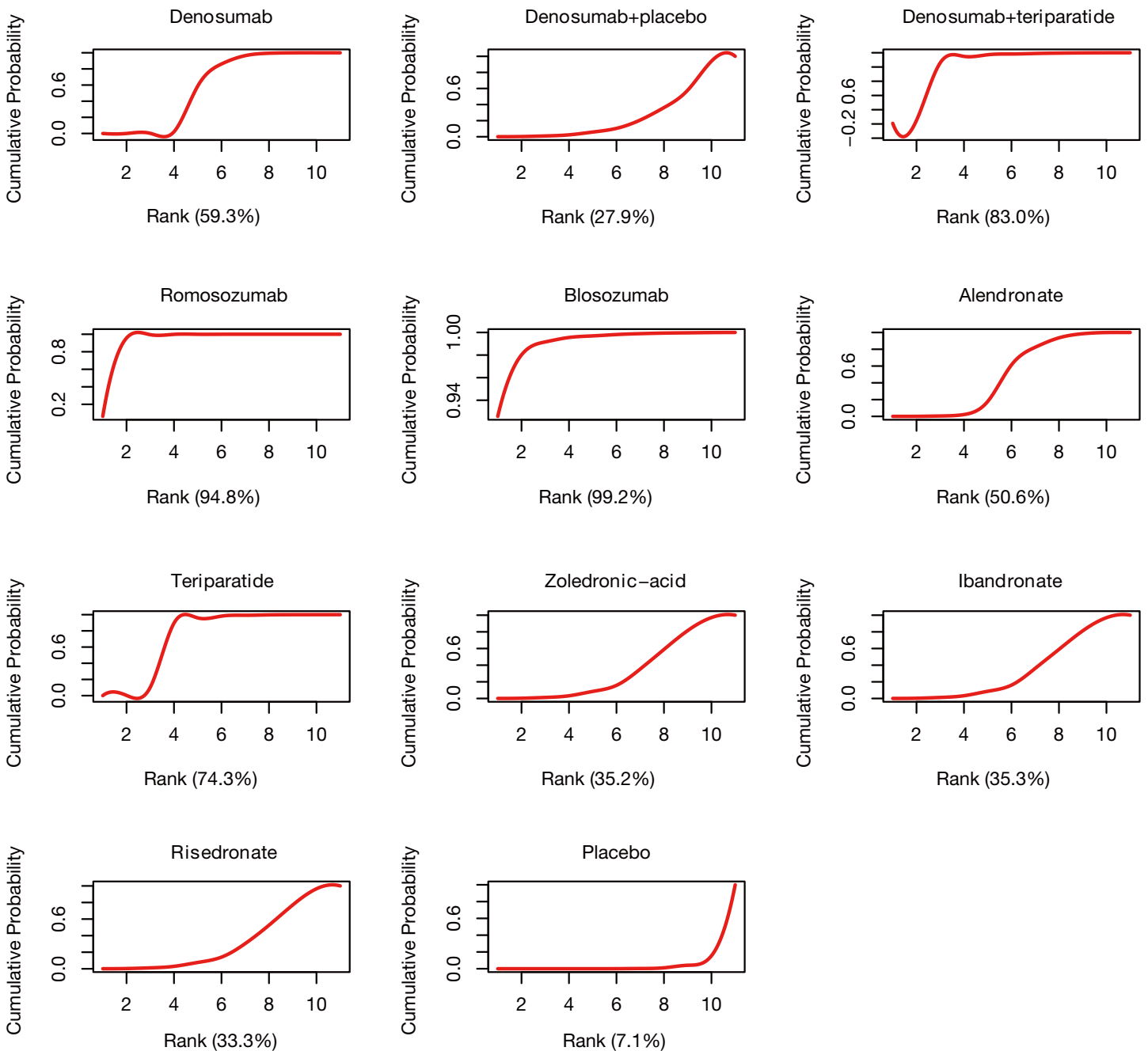

Figure 5 Cumulative ranking probability diagram and the corresponding SUCRA value of each agent. SUCRA, surface under the cumulative ranking area.

\section{Conclusions}

This NMA demonstrated that McAbs outperformed the conventional chemical drugs. Blosozumab was considered the preferred agent for improving lumbar spine BMD. We paved the way for clinical intervention strategies concerning novel targeted drugs. Nevertheless, more high-quality RCTs are necessary for more comprehensive evaluations and treatments to further our conclusions.

\section{Acknowledgments}

Funding: This research was funded by Jiangsu Provincial 'Qinglan Talents Project', Science and Education Promotion Project of JSCDC (JKRC2016015) and Jiangsu
Medical Innovation Team (CXTDA2017029).

\section{Footnote}

Reporting Checklist: The authors have completed the PRISMA reporting checklist. Available at http://dx.doi. org/10.21037/apm-20-1294

Conflicts of Interest: All authors have completed the ICMJE uniform disclosure form (available at http://dx.doi. org/10.21037/apm-20-1294). The authors have no conflicts of interest to declare.

Ethical Statement: The authors are accountable for all 
aspects of the work in ensuring that questions related to the accuracy or integrity of any part of the work are appropriately investigated and resolved.

Open Access Statement: This is an Open Access article distributed in accordance with the Creative Commons Attribution-NonCommercial-NoDerivs 4.0 International License (CC BY-NC-ND 4.0), which permits the noncommercial replication and distribution of the article with the strict proviso that no changes or edits are made and the original work is properly cited (including links to both the formal publication through the relevant DOI and the license). See: https://creativecommons.org/licenses/by-nc-nd/4.0/.

\section{References}

1. Rachner TD, Khosla S, Hofbauer LC. Osteoporosis: now and the future. Lancet 2011;377:1276-87.

2. Brown C. Osteoporosis: Staying strong. Nature 2017;550:S15-S17.

3. Li X, Ominsky MS, Warmington KS, et al. Sclerostin antibody treatment increases bone formation, bone mass, and bone strength in a rat model of postmenopausal osteoporosis. J Bone Miner Res 2009;24:578-88.

4. Black DM, Rosen CJ. Clinical Practice. Postmenopausal Osteoporosis. N Engl J Med 2016;374:254-62.

5. Cheng C, Wentworth K, Shoback DM. New Frontiers in Osteoporosis Therapy. Annu Rev Med 2020;71:277-88.

6. Crandall CJ, Newberry SJ, Diamant A, et al. Comparative effectiveness of pharmacologic treatments to prevent fractures: an updated systematic review. Ann Intern Med 2014;161:711-23.

7. Harrison C. Trial watch: Bone-building antibody outshines current treatments in osteoporosis trial. Nat Rev Drug Discov 2014;13:248.

8. Khosla S. Personalising osteoporosis treatment for patients at high risk of fracture. Lancet Diabetes Endocrinol 2019;7:739-41.

9. Lewiecki EM. New targets for intervention in the treatment of postmenopausal osteoporosis. Nat Rev Rheumatol 2011;7:631-8.

10. Makras P, Delaroudis S, Anastasilakis AD. Novel therapies for osteoporosis. Metabolism 2015;64:1199-214.

11. Onuora S. Gene therapy counteracts bone loss in osteoporosis. Nat Rev Rheumatol 2019;15:513.

12. Qaseem A, Forciea MA, McLean RM, et al. Treatment of Low Bone Density or Osteoporosis to Prevent Fractures in Men and Women: A Clinical Practice Guideline Update
From the American College of Physicians. Ann Intern Med 2017;166:818-39.

13. Chen WC, Lin EY, Kang YN. Efficacy and safety of elcatonin in postmenopausal women with osteoporosis: a systematic review with network meta-analysis of randomized clinical trials. Osteoporos Int 2019;30:1723-32.

14. Yang L, Kang N, Yang JC, et al. Drug efficacies on bone mineral density and fracture rate for the treatment of postmenopausal osteoporosis: a network meta-analysis. Eur Rev Med Pharmacol Sci 2019;23:2640-68.

15. An Q, Liu Z. Comparative efficacy and safety of combination therapies for advanced melanoma: a network meta-analysis. BMC Cancer 2019;19:43.

16. Cheng J, Cai M, Shuai X, et al. Systemic therapy for previously treated advanced gastric cancer: A systematic review and network meta-analysis. Crit Rev Oncol Hematol 2019;143:27-45.

17. Kondo Y, Goto A, Noma H, et al. Effects of Coffee and Tea Consumption on Glucose Metabolism: A Systematic Review and Network Meta-Analysis. Nutrients 2018;11:48.

18. Yang P, Wang Q, Xie C, et al. Efficacy and Safety of Agents in IgA Nephropathy: An Update Network MetaAnalysis. Kidney Blood Press Res 2018;43:1890-7.

19. Yang P, Zou H, Xiao B, et al. Comparative Efficacy and Safety of Therapies in IgA Nephropathy: A Network Meta-analysis of Randomized Controlled Trials. Kidney Int Rep 2018;3:794-803.

20. McClung MR, Lewiecki EM, Cohen SB, et al. Denosumab in postmenopausal women with low bone mineral density. N Engl J Med 2006;354:821-31.

21. Kendler DL, Roux C, Benhamou CL, et al. Effects of denosumab on bone mineral density and bone turnover in postmenopausal women transitioning from alendronate therapy. J Bone Miner Res 2010;25:72-81.

22. Bolognese MA, Teglbjaerg CS, Zanchetta JR, et al. Denosumab significantly increases DXA BMD at both trabecular and cortical sites: results from the FREEDOM study. J Clin Densitom 2013;16:147-53.

23. Recknor C, Czerwinski E, Bone HG, et al. Denosumab compared with ibandronate in postmenopausal women previously treated with bisphosphonate therapy: a randomized open-label trial. Obstet Gynecol 2013;121:1291-9.

24. Tsai JN, Uihlein AV, Lee H, et al. Teriparatide and denosumab, alone or combined, in women with postmenopausal osteoporosis: the DATA study randomised trial. Lancet 2013;382:50-6.

25. Roux C, Hofbauer LC, Ho PR, et al. Denosumab 
compared with risedronate in postmenopausal women suboptimally adherent to alendronate therapy: efficacy and safety results from a randomized open-label study. Bone 2014;58:48-54.

26. Koh JM, Chung DJ, Chung YS, et al. Assessment of Denosumab in Korean Postmenopausal Women with Osteoporosis: Randomized, Double-Blind, PlaceboControlled Trial with Open-Label Extension. Yonsei Med J 2016;57:905-14.

27. Miller PD, Pannacciulli N, Brown JP, et al. Denosumab or Zoledronic Acid in Postmenopausal Women With Osteoporosis Previously Treated With Oral Bisphosphonates. J Clin Endocrinol Metab 2016;101:3163-70.

28. McClung MR, Lippuner K, Brandi ML, et al. Effect of denosumab on trabecular bone score in postmenopausal women with osteoporosis. Osteoporos Int 2017;28:2967-73.

29. McClung MR, Grauer A, Boonen S, et al. Romosozumab in postmenopausal women with low bone mineral density. N Engl J Med 2014;370:412-20.

30. Genant HK, Engelke K, Bolognese MA, et al. Effects of Romosozumab Compared With Teriparatide on Bone Density and Mass at the Spine and Hip in Postmenopausal Women With Low Bone Mass. J Bone Miner Res 2017;32:181-7.

31. Ishibashi H, Crittenden DB, Miyauchi A, et al. Romosozumab increases bone mineral density in postmenopausal Japanese women with osteoporosis: A phase 2 study. Bone 2017;103:209-15.

32. Keaveny TM, Crittenden DB, Bolognese MA, et al. Greater Gains in Spine and Hip Strength for Romosozumab Compared With Teriparatide in Postmenopausal Women With Low Bone Mass. J Bone Miner Res 2017;32:1956-62.

33. Recker RR, Benson CT, Matsumoto T, et al. A randomized, double-blind phase 2 clinical trial of

Cite this article as: Jiang Q, Zhang F, Zhu B, Zhang H, Han L, Liu X. Comparative efficacy between monoclonal antibodies and conventional drugs in postmenopausal women with osteoporosis: a network meta-analysis. Ann Palliat Med 2021;10(2):1693-1702. doi: 10.21037/apm-20-1294 blosozumab, a sclerostin antibody, in postmenopausal women with low bone mineral density. J Bone Miner Res 2015;30:216-24.

34. Andreopoulou P, Bockman RS. Management of postmenopausal osteoporosis. Annu Rev Med 2015;66:329-42.

35. Kalyan S, Sharma R, Torka P, et al. Romosozumab Treatment in Postmenopausal Osteoporosis. N Engl J Med 2017;376:395-6.

36. Ke HZ, Richards WG, Li X, et al. Sclerostin and Dickkopf-1 as therapeutic targets in bone diseases. Endocr Rev 2012;33:747-83.

37. Kondegowda NG, Fenutria R, Pollack IR, et al. Osteoprotegerin and Denosumab Stimulate Human Beta Cell Proliferation through Inhibition of the Receptor Activator of NF-kappaB Ligand Pathway. Cell Metab 2015;22:77-85.

38. Reid IR. Targeting Sclerostin in Postmenopausal Osteoporosis: Focus on Romosozumab and Blosozumab. BioDrugs 2017;31:289-97.

39. Zha L, He L, Liang Y, et al. TNF-alpha contributes to postmenopausal osteoporosis by synergistically promoting RANKL-induced osteoclast formation. Biomed Pharmacother 2018;102:369-74.

40. Papapoulos SE. Bone: Romosozumab-getting there but not quite yet. Nat Rev Endocrinol 2016;12:691-2.

41. Rosen CJ. Romosozumab-Promising or Practice Changing? N Engl J Med 2017;377:1479-80.

42. Collison J. Osteoporosis: Discontinuing denosumab discouraged. Nat Rev Rheumatol 2017;13:571.

43. Compston J. Safety of long-term denosumab therapy for osteoporosis. Lancet Diabetes Endocrinol 2017;5:485-7.

44. Eastell R, Szulc P. Use of bone turnover markers in postmenopausal osteoporosis. Lancet Diabetes Endocrinol 2017;5:908-23. 
Random sequence generation

Allocation concealmen
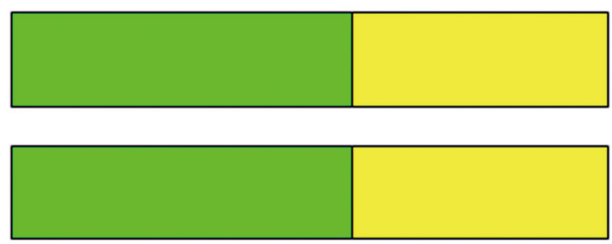

Blinding of participants and personne

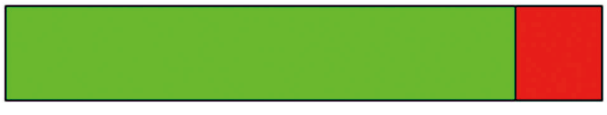

Blinding of outcome assessment

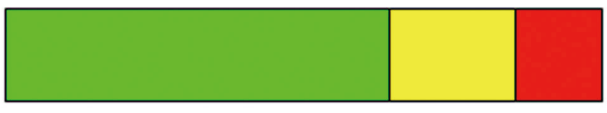

Incomplete outcome dat

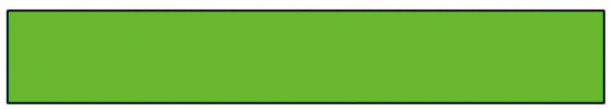

Selective reporting

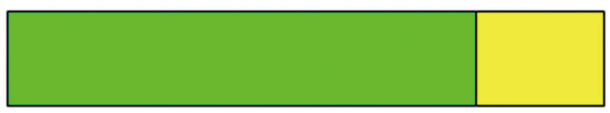

Other sources of bias

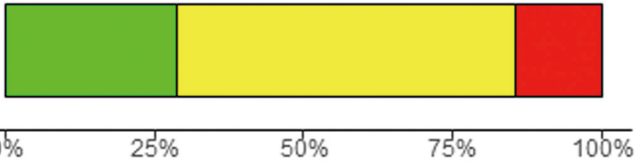

Low risk of bias

High risk of bias

\section{unclear}

Figure S1 Risk of bias summary.

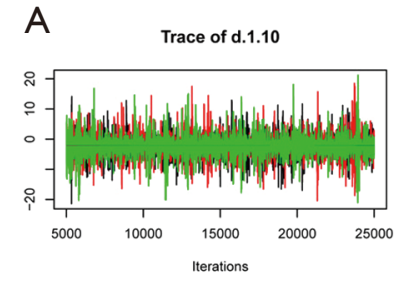

Trace of d.1.11
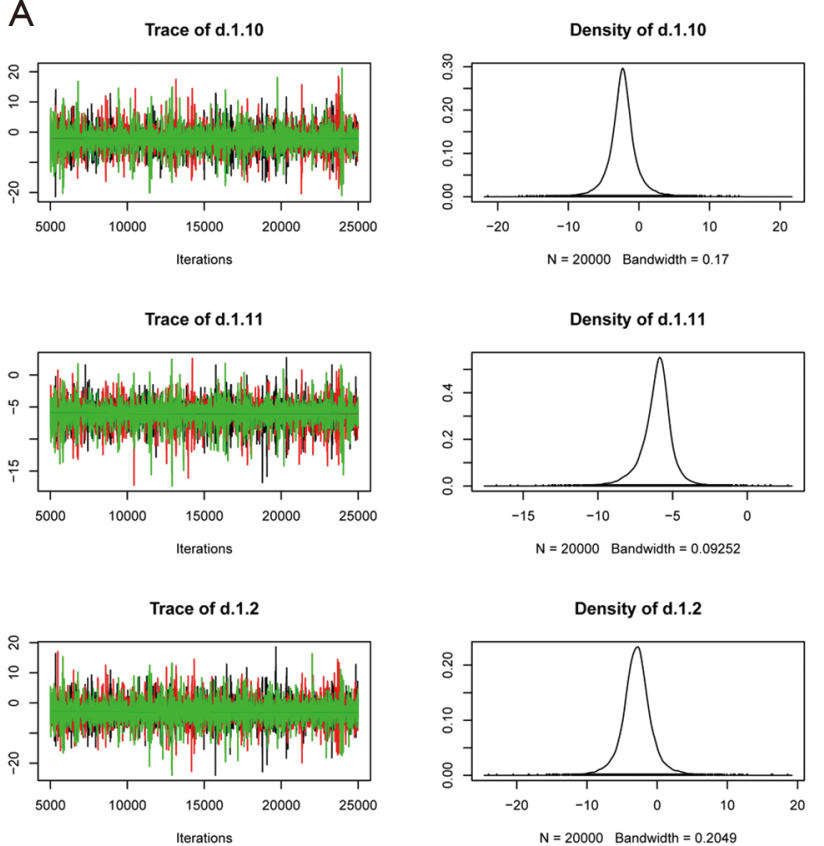

C
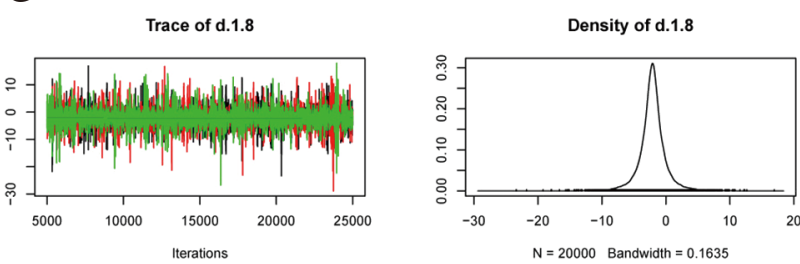

Trace of d.1.9

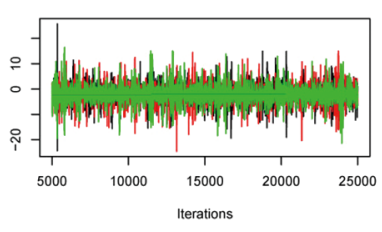

Trace of d.11.4

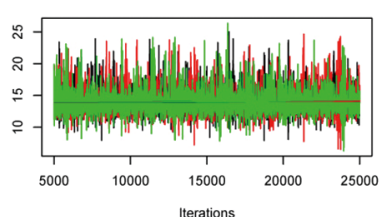

B

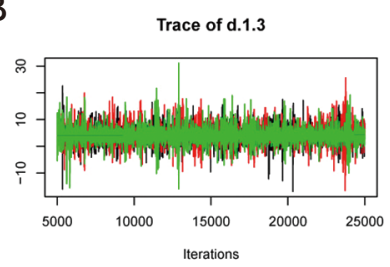

Trace of d.1.6

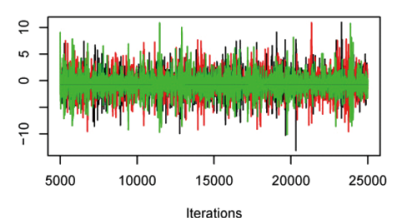

Trace of d.1.7

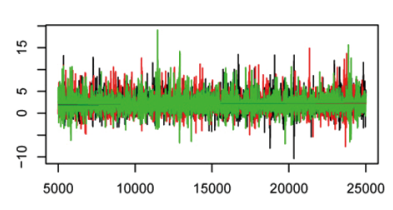

Iterations

D
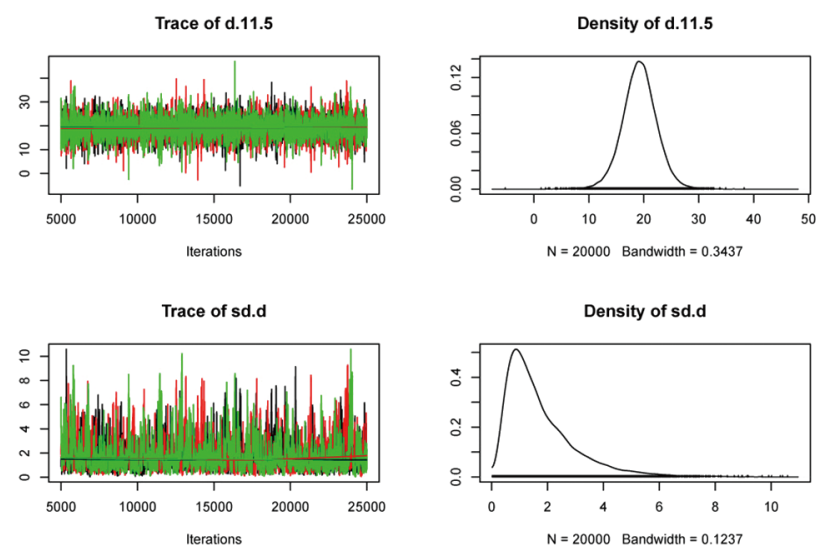

Figure S2 Trace-density graphs with different iteration instances. 


\section{d.1.10}

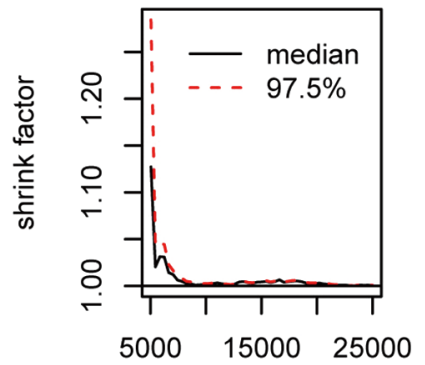

last iteration in chain

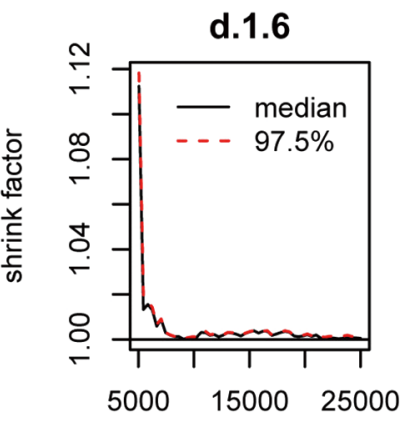

last iteration in chain d.1.11

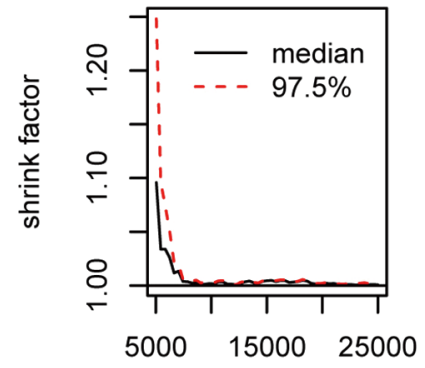

last iteration in chain

d.1.7

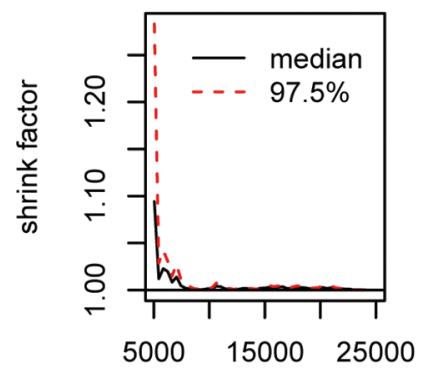

last iteration in chain d.1.2

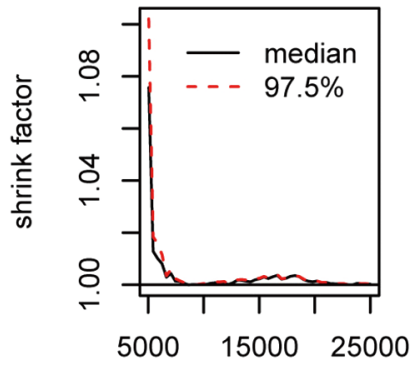

last iteration in chain

\section{d.1.8}

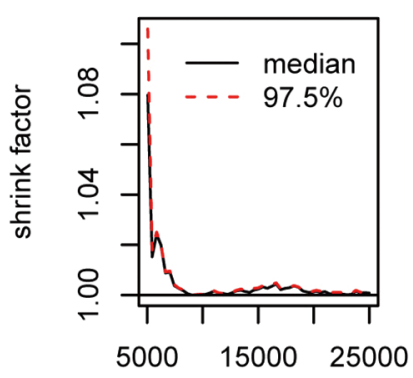

last iteration in chain d.1.3

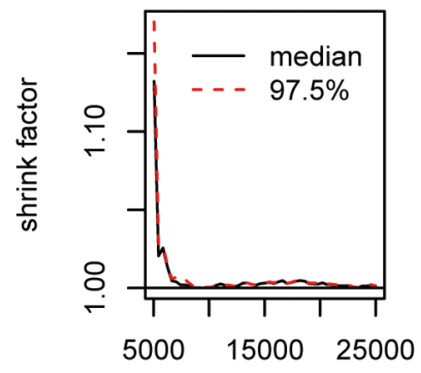

last iteration in chain

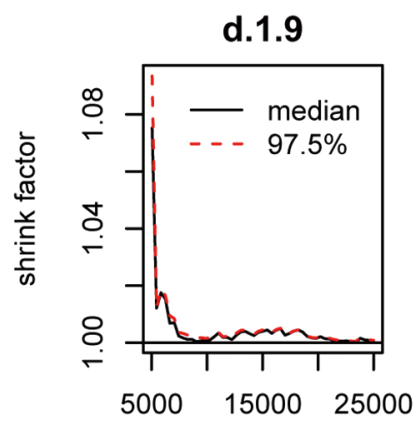

last iteration in chain

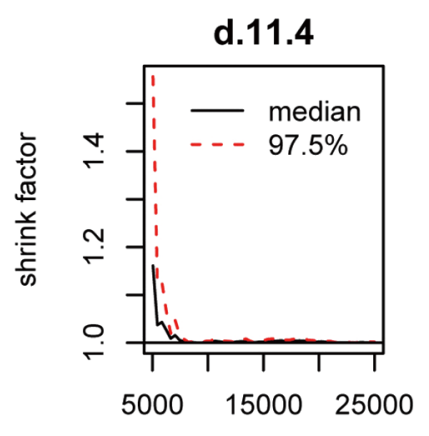

last iteration in chain

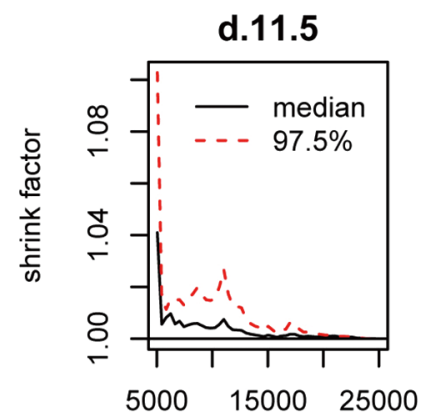

last iteration in chain

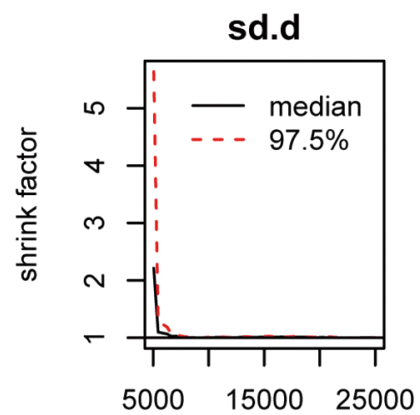

last iteration in chain

Figure S3 Brooks-Gelman-Rubin diagnostic diagrams with different iterations. 


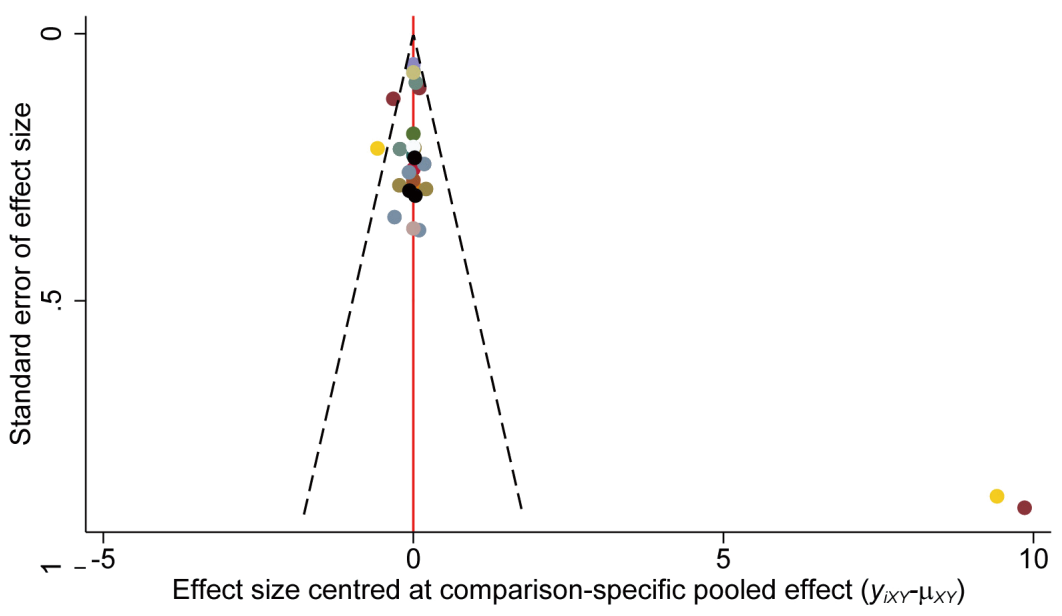

\begin{tabular}{|lllllll|}
\hline 1 vs 10 & $\bullet 1$ vs 11 & $\bullet 1$ vs 2 & $\bullet 1$ vs 3 & $\bullet 1$ vs 6 & $\bullet 1$ vs 7 \\
1 vs 8 & 1 vs 9 & $\bullet 3$ vs 7 & $\bullet 4$ vs 11 & $\bullet 4$ vs 6 & $\bullet 4$ vs 7 \\
5 vs 11 & 6 vs 11 & 6 vs 7 & $\bullet 7$ vs 11 &
\end{tabular}

Figure S4 Funnel plots assessing publication bias. Asymmetry patterns indicate the presence of potentially significant publication bias.

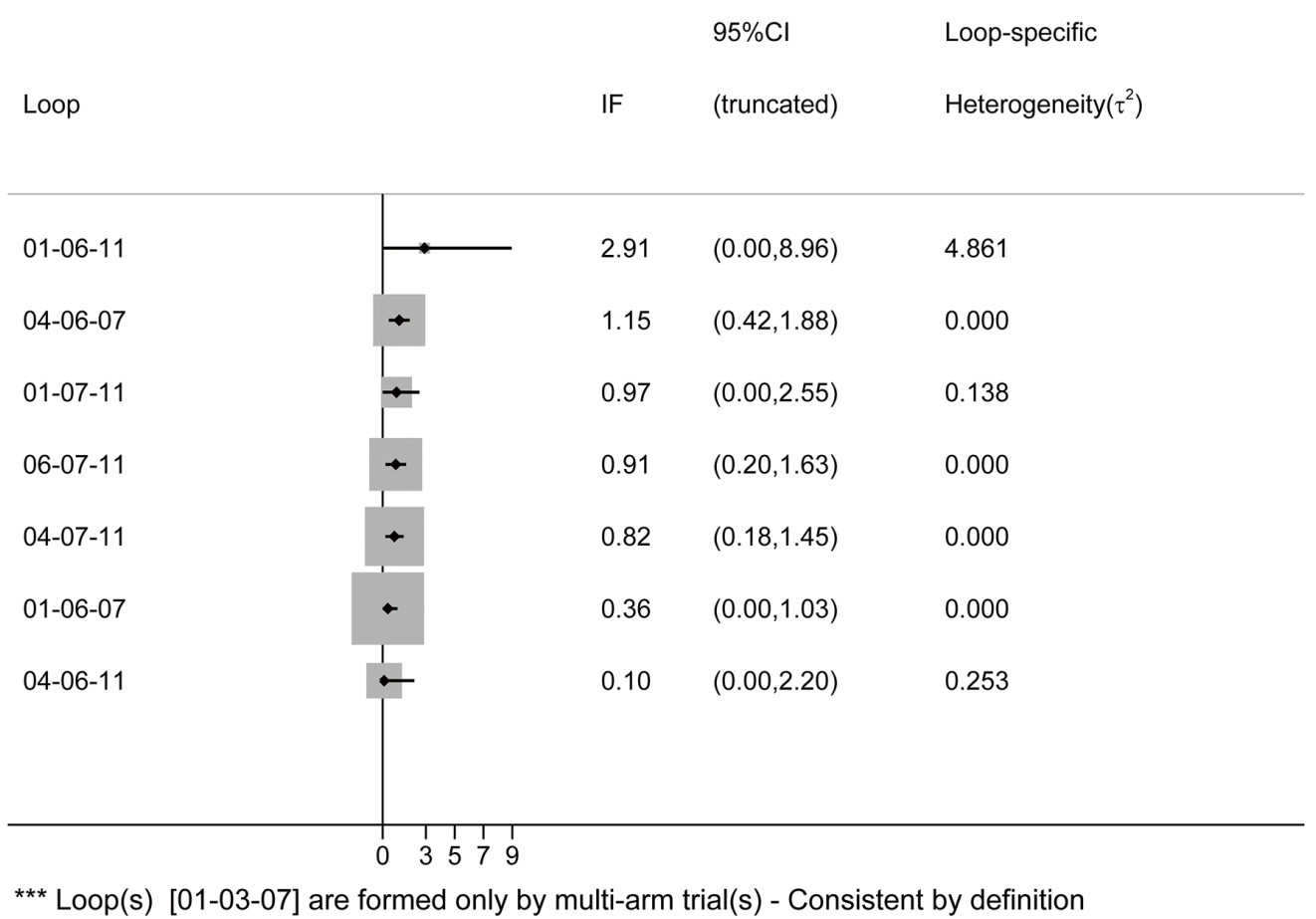

Figure S5 Inconsistency test results using the method of moments. 